\title{
Methicillin Resistant Staphylococcus aureus nasal carriage and associated factors in a rural tertiary hospital in Eastern Uganda: A prospective cross- sectional study
}

Nobert Thembo ( $\nabla$ nobertt50@gmail.com )

Mbale Regional Referral Hospital

George Masifa

Mbale Regional Referral Hospital

Gerald Kamugisha

Mbarara University of Science and Technology

Robinah Nabitaka

Mbarara University of Science and Technology

Benjamin Akais

Mbarara University of Science and Technology Faculty of Medicine

Peter Olupot-Olupot

Mbale Regional Referral Hospital

Rita Muhindo

Mbale Regional Referral Hospital

Edward Kiyonga

Mbarara University of Science and Technology Faculty of Medicine

Lucas Ampaire

Mbarara University of Science and Technology

Research article

Keywords: Nasal carriage, S aureus, Methicillin Resistance

Posted Date: March 11th, 2020

DOI: https://doi.org/10.21203/rs.2.13651/v3

License: (c) (i) This work is licensed under a Creative Commons Attribution 4.0 International License.

Read Full License 


\section{Abstract}

Background: Asymptomatic carriage of Methicillin-Resistant Staphylococcus aureus (MRSA) can predispose the host to a wide array of infections that can be difficult to treat due to antibiotic resistance. To inform public health strategies, the study sought to describe MRSA nasal carriage frequencies and the associated factors concerning nasal carriage among patients attending Mbale Regional Referral Hospital (MRRH). Methods: Nasal swabs were obtained from consented (aged $>15 y e a r s$ ) participants presenting to the hospital for medical care between January and April 2018 [L1] . Direct Culture of swabs was performed on blood agar and then incubated at $37^{\circ} \mathrm{C}$ for 24 hours. Identification of $\mathrm{S}$. aureus was done using conventional biochemical tests. Phenotypic screening and confirmation of MRSA was done using cefoxitin disc (30 $\mathrm{gg})$ test and MICs on the Phoenix M50 instrument respectively. Patient demographic characteristics and the MRSA nasal carriage risk factors were collected using a pre-tested questionnaire. Results: Overall, majority of the participants were in-patients $(138,63.3 \%)$ with the proportions of both females and males among the participants being 154/218 (70.6\%) and 64/218 (29.3\%) respectively. Mean age for both female and male participants was $40.16(\mathrm{SD} \pm 17.04)$ years respectively. $S$. aureus nasal carriage rate among the participants was 22.9\% (50/218), with $57.9 \%(29 / 50)$ of the harboured strains phenotypically expressing methicillin resistance ( mecA mediated). Phenotypic co-expression with i nducible clindamycin resistance and vancomycin resistance was displayed in $45.5 \%(23 / 50)$ and $2 \%$ $(1 / 50)$ of the studied isolates respectively. Colonisation with MRSA did not show any significant relationship with all the studied factors. Conclusion : There was a moderate S. aureus nasal carriage among the participants in Mbale Regional Referral Hospital with a highly noted phenotypic expression of methicillin resistance among the isolated $\mathrm{S}$. aureus strains. The studied factors were not significantly associated with the rate of MRSA nasal carriage. For surveillance purposes to combat future outbreaks, there is a need to do a larger study to better draw generalizable conclusions of carriage in the population.

[L1] This is an important statement. I suggest that we reinstatate it if we are still in recommended word count

\section{Background}

S. aureusis among the most significant human bacterial pathogens worldwide (1), with MRSA currently being the most common antibiotic-resistant strain in most of the hospital and community settings (2)(3). $S$. aureus is also the second leading cause of nosocomial bacteremia (4) and has been highlighted as a priority organism of interest by the WHO (5). Asymptomatic nasal carriage of MRSA can predispose the host to a wide array of infections that would pose a challenge in the management of the cases in the current era of antibiotic resistance (6)(7).

The magnitude of MRSA remains greatly unknown in developing countries yet surveillance systems to guide interventions require expertise and resources, which are inadequate(8). Studies conducted elsewhere by Conceiçao et al., and Aiken et al., documented MRSA prevalence rates of $26.9 \%$ and $7.0 \%$ respectively (9)(10). However, in resource-limited settings, not much has been done to explore on MRSA nasal carriage as reported by Bebell et al., 2016 and yet MRSA is currently recognized as the leading 
cause of hospital-acquired infections (11). Empirical and inappropriate use of antibiotics, multiplepathology and use of invasive devices have attributed to a high rate of MRSA hence extended hospital duration and high treatment costs among patients (12). Documented reports about Methicillin resistance in WHO regions an exceeding $20 \%$ proportion with the highest recorded being $80 \%$ in some regions (13). In Africa, heterogeneity has been reported in the MRSA prevalence rates both intra-country and intercountry (14). Data collected from nine African countries indicates that MRSA prevalence rates range between $12 \%$ and $80 \%$ (15)(16). In East Africa and Uganda in particular has documented high proportions of between 31.5 to $42 \%$ within patients and healthcare professionals (17)(18).

Identifying the source, reservoirs and vectors for the spread of antibiotic-resistant bacteria poses significant challenges in tracking antimicrobial resistance. The patient's carried endogenous microflora may all play a vital role in the cause of nosocomial infections (19)(20). In a study conducted on surgical wound infections in the hospital indicated that $65.9 \%$ of the cases were caused by MRSA strains (21). There are currently no documented reports on nasal colonization and carriage rates of MRSA among patients and associated carriage factors in Mbale Regional Referral Hospital. With most patients coming from different settings, our study aimed to determine the prevalence of $S$. aureus and MRSA nasal colonization, and further identify the risk factors associated with asymptomatic carriage of MRSA.

\section{Methods}

\section{Study design}

This was a descriptive cross-sectional study carried out between January and April 2018 at Mbale Regional Referral Hospital on both in and Outpatients.

\section{Study area}

The study was conducted at both the Outpatient and In-patient departments (wards) of Mbale Regional Referral Hospital located in Mbale municipality. The hospital serves as both a regional referral and a tertiary teaching hospital for the medical school of Busitema University and School of clinical officers. It has a bed capacity of 400 and serves a population of approximately 4 million people.

\section{Target population}

The study targeted all patients seeking medical care at Mbale RRH who are asymptomatic carriers of MRSA. These included those from the inpatient (medical, surgical and maternity wards) and outpatient departments.

\section{Inclusion Criteria}

We included Patients aged 15 years and above, seeking medical care at either the In-patient or the Outpatient department at MRRH and had consented to take part in the study. Written informed consent for children aged 16 and 15 years was sought from their parents or guardians. 


\section{Sample size and recruitment of study participants}

The sample size estimation was calculated using Kish Leslie formula. Consent was sought prior to recruiting of the participants. A cluster random sampling technique was used whereby participants were selected randomly with respect to the two broad clusters i.e. OPD and in-patients to ensure equal representative sampling. A questionnaire was administered through interviewing of participants to capture both demographic factors and predictor factors for MRSA nasal carriage.

\section{Sample collection and transportation}

From each participant, specimens for $S$. aureus culture were collected from both the anterior nares using sterile broth moistened dry swabs. The swabs were immediately placed into a tube with Cary-Blair transport media (BBL ${ }^{\mathrm{TM}}, \mathrm{BD}$ bioscience), labelled with the participants' study number, initials, date and time of sample collection. Samples were immediately delivered to the Clinical Microbiology Laboratory, Mbale RRH for processing.

\section{Isolation and identification of $S$. aureus}

The swabs were first inoculated on Blood Agar and incubated at $37^{\circ} \mathrm{C}$ aerobically for 24 hours after which plate reading was done. Gram staining was performed on discrete colonies displaying the culture characteristics of $S$. aureus to confirm for Gram-positive cocci in clusters. A series of conventional biochemical tests such as catalase test using $3 \%$ hydrogen peroxide, bound and free coagulase using reconstituted rabbit lyophilized plasma (Remel, Europe, Itd. Dartford, Kent DA2 6PT, UK) were performed to identify the $S$. aureus. Further confirmation was done by sub-culturing of the isolated colonies of $S$. aureus onto Mannitol Salt Agar (MSA, Oxoid, CM0085. Thermo-scientific, US) and incubated at $37^{\circ} \mathrm{C}$ for 24 hours aerobically. After 24 hours, the plates were examined for growth with interest in mannitol fermenting colonies that appeared as yellow colonies, measuring 1-2 mm in diameter and slightly raised.

\section{Preservation of the Isolates}

By use of a sterile pre-flamed wire loop, pure growing colonies were scraped off the Blood agar purity plates and suspended in $1 \mathrm{ml}$ of $15 \%$ glycerol broth the frozen at $-80^{\circ} \mathrm{C}$ till needed for MRSA phenotypic testing.

\section{Phenotypic detection for MRSA}

Agar disk diffusion (Kirby Bauer) technique on $4 \%$ sodium chloride Mueller Hinton Agar (BBL ${ }^{T M}, B D$ ) was employed. The inoculum was prepared by picking distinct colonies from a fresh pure loon culture on Blood agar and suspended into $5 \mathrm{mls}$ of a $0.85 \%$ saline water to make a bacterial suspension. The suspension was vortexed for 15 seconds and the turbidity adjusted visually by adding sufficient saline water to achieve a 0.5 McFarland standard. A sterile cotton swab was dipped into the suspension, rotated several times and the excess fluid removed by pressing on the sidewalls of the tube. The dried surface of the agar was evenly streaked with the sterile swab aseptically rotating the plate at $60^{\circ}$ to ensure uniform 
distribution. A cefoxitin disc ( $30 \mu \mathrm{g}$, HIMedia, Mumbai, India) was placed on the surface of the inoculated plate using sterile forceps. The setup was incubated at $37^{\circ} \mathrm{C}$ for 24 hours aerobically. The plates were read after 24 hours of incubation and the zone diameters of inhibition were measured using a Vernier calliper. The measured zones of inhibition were recorded and interpretations were done following the interpretive cut-offs (MRSA $\leq 21 \mathrm{~mm}$ and MSSA $\_22 \mathrm{~mm}$ ) as per the Clinical Laboratory Standard Institute (CLSI) guidelines (22). The positively screened isolates were subjected to cefoxitin MICs $(>8 \mathrm{mg} / \mathrm{mL}$ ) to confirm for MRSA carriage on the Phoenix M50 instrument using PMIC panels (BD) for confirmation.

\section{Data Analysis}

Data analysis was done using Stata Corp. version 13. Descriptive statistics including proportions, means were used to describe the participants and to determine prevalence. We considered a univariate analysis at a level of significance of 0.2 to describe the categorical variables by sex and obtain baseline characteristics. The $P$-value was adjusted to allow for multiple logistic regression. Associations were generated using Odds ratio at $95 \% \mathrm{Cl}$ and a $P$-value of $\leq 0.05$. We performed a logistic regression to determine any association between the factors collected as predictors and MRSA nasal carriage as the outcome.

\section{Quality Control}

Standard Operating procedures were followed, aseptic transfer techniques were performed, reagents were kept at $2-8^{\circ} \mathrm{C}$, and the questionnaire was pretested before the commencement of the study. $S$. aureus ATCC 25923 was used as the positive control strain for the identification of biochemical tests and susceptibility tests on $4 \%$ sodium chloride Muller Hinton Agar. Commercially available control strain of $S$. aureus ATCC 700699 was used as a live positive control for MRSA on the PMIC panels.

\section{Results}

\section{Demographic characteristics of patients}

The study recruited 218 participants aged 15 years and above presenting to Mbale Regional Referral Hospital for medical care. Majority of the participants were in-patients 138/218 (63.3\%) with the proportions of both females and males among the participants being 154/218 (70.6\%) and 64/218 (29.3\%) respectively. Mean age for both female and male participants was $40.16(S D \pm 17.04)$ years respectively as shown in table 1 below.

\section{The proportion of patients with S. aureus and MRSA nasal carriage attending Mbale Regional Referral Hospital}


The prevalence rate for $S$. aureus nasal carriage among the participants was $22.9 \%$ (50/218) as indicated in table 2 below. Proportions for MRSA nasal carriage among the participants were found to be $13.3 \%$ (29/218) and 9.63\% (21/218) of whom were colonized with MSSA (table 3).

\section{Distribution of Methicillin resistance among S. aureus Isolates.}

Of the 50-studied S. aureus isolates, 57.9\% (29/50) of the isolates were found to be phenotypically expressing methicillin resistance (mecA mediated) while 42.1\% (21/50) were methicillin sensitive as seen in figure 1. Co-expression of MRSA with inducible clindamycin resistance and vancomycin resistance was observed among $45.5 \%(23 / 50)$ and $2 \%(1 / 50)$ respectively.

\section{Multivariate analysis of studied factors for association with Methicillin Resistant $S$. aureus nasal colonization.}

Colonization with Methicillin Resistant $S$. aureus was not significantly associated with age (OR=0.9825889; 95\% Cl=0.9612351-1.004417, $P$-value=0.117), history of hospitalization (OR=1.156944; $95 \% \mathrm{Cl}=0.5689258-2.352712 ; P$-value $=0.687)$, contact with animals $(\mathrm{OR}=1.141084 ; 95 \% \mathrm{Cl}=0.8069104-$ 1.613653; $P$-value=0.455), antibiotic use (OR=0.7516896; $95 \% \mathrm{Cl}=0.3804775-1.485974 ; P$-value $=0.411)$ and HIV status (OR=0.7215959; 95\% $\mathrm{Cl}=0.2871594-2.107922 ; P$-value=0.551).

\section{Discussion}

\section{Prevalence of $S$. aureus nasal carriage}

Results from this study indicated that the nasal carriage rates for $S$. aureus among the participants was $22.9 \%$, a rate significantly higher than that reported from studies from Thika Hospital, Kenya (8.9\%), Nigeria (18.3\%), and China (2.4\%) (23)(24)(25). Studies conducted elsewhere by Bebell et al, Ouedraogo et al, Moniri et al and Ateba et al revealed slightly higher rates of $28.5 \%, 29.1 \%, 32.9 \%, 38 \%$ and $29 \%$ respectively compared to the finding in our study $(11)(26)(27)(28)$. However, our finding was similar to reports from North Germany by Mehraj et al., Northwest Ethiopia and Italy, which documented $S$. aureus nasal carriage rates of $21.9 \%$ and $23 \%$ respectively (29)(30). The difference in the various findings would have been attributed to the sample size used giving the different studies a better power to detect the $S$. aureus nasal carriage. In addition, the studies were conducted in different geographical regions and the regions might have differing antibiotic usage practices hence differences in the carriage bio-films.

\section{MRSA carriage Prevalence and associated carriage risk factors}

In our study, we determined the prevalence of MRSA and MSSA among randomly selected participants who presented to the hospital for medical care. Our study documented an overall prevalence rate of $13.3 \%$ (29/218) for MRSA among the participants who were included in the study. However, we noted a high 57.9\% (29/50) methicillin resistance expression among the carried $S$. aureus strains. The finding for the overall carriage rate (13.3\%) among the participants was lower than the reported prevalence of $46 \%$ from Mulago hospital, Kampala, Uganda (18). However, our finding was higher than a prevalence rate of 
2.8\% reported by Bebell et al from Mbarara Hospital, Uganda (11). Our observed high (57.9\%) methicillin resistance expression among the isolated $S$. aureus strains was contrary to reports from three regional hospitals in Tanzania that is Muhimbili National Hospital (10.5\%), Mwananyamala and Amana regional hospitals (24.7\%) (31)(32). An attribution to the high MRSA carriage could have been due to the high numbers of in-patients who were included in the study and duration of hospital stay could have had an effect of acquisition. In addition, the choice of the participants included could have affected the prevalence since our study did not consider the children age group. Among the MRSA strains identified, $45.5 \%(23 / 50)$ and $2 \%(1 / 50)$ phenotypically co-expressed inducible clindamycin resistance $\left(M L S_{B}\right.$ resistance marker) and vancomycin resistance respectively. A study by Mwanbi et al conducted in Uganda on clinical isolates reported a closely similar prevalence rate $(36 \%)$ of clindamycin resistance (33). Resistance to clindamycin would have been due to an increased use of the drug for patient management in the region.

In our study, colonization by MRSA did not indicate any significant statistical association with all the tested variables. However, we observed different levels of consistency with the findings of previous studies. Our study indicated that antibiotic usage did not increase the likelihood of colonization by MRSA (OR=0.7516896; 95\% Cl=0.3804775-1.485974; $P$-value=0.411), while HIV status was found to have no effect on the nasal carriage rate even with low immunity (OR=0.7215959; $95 \% \mathrm{Cl}=0.2871594-2.107922 ; P$ value $=0.551$ ). The statistical association findings by Bhattacharya et al that reported no association with immune status or recent antibiotic use (34), were similar to the findings in our study. Nonetheless, the no association with all the variable factors might be attributed to the difference in the characteristics and varied geographical location for the participants. The study had a limitation with the small sample size, which could have inferred a less power and generalization of results for MRSA carriage in the population.

\section{Conclusion}

Our study revealed that there was a moderate $S$. aureus nasal carriage among the participants but with high methicillin resistance expressed (57.9\%) among the isolated strains. Assessment of the factors with MRSA nasal carriage rates revealed that none of the factors significantly influenced carriage. The study has been the first in Mbale, Uganda to provide baseline data on $S$. aureus and MRSA nasal carriage. Therefore, there is need to do a larger extensional surveillance to understand the drivers and transmission patterns for $S$. aureus and MRSA within the hospital and community. However, the study had some limitations and in particular, there is still need to further phenotypically or genotypically assess the isolates using PBP2a agglutination assay or by PCR respectively to better understand the carriage. There is also need to evaluate a link between hospital acquired infection, carriage and possibility of zoonotic transmission.

\section{Abbreviations}

MCRI: Mbale Clinical Research Institute 
MRSA: Methicillin-Resistant Staphylococcus aureus

MRRH: Mbale Regional Referral Hospital

WHO: World Health Organization

OPD: Out Patient Department

Cl: Confidence Interval

CLSI: Clinical Laboratory Standard Institute

ATCC: America Type Culture Control

SD: Standard Deviation

HIV: Human Immune Virus

MSSA: Methicillin Susceptible Staphylococcus aureus

OR: Odds ratios

MRRH-REC: Mbale Regional Referral Hospital Research Ethics Committee

CSV: Comma Separated Version Files

CoNS: Coagulase Negative Staphylococcus

N/A: Not Applicable.

\section{Declarations}

\section{Acknowledgements}

We are grateful to the study participants and hospital management of Mbale regional referral hospital especially theatre staff for their cooperation and technical guidance. We also thank the academic staff of Mbarara University of Science and Technology, Faculty of Medicine for their tremendous mentorship.

\section{Ethics approval and consent to participate}

Ethical approval was obtained from the Faculty of Medicine Research Committee at Mbarara University of Science and Technology (Ref: DMS/6) and Mbale Regional Referral Hospital Ethics Review Committee (Ref: MRRH-REC-IN-COM 110/2017). Written informed consent was obtained from the patients who had satisfied the study eligibility criteria for inclusion. The consenting process was done in a private room and all the participants were allocated identification numbers to ensure the confidentiality of the participants. 


\section{Consent to publish}

Not applicable

\section{Availability of data and materials}

The data sets in comma separated version files (CSV) are available and have been attached as supplementary information files.

\section{Competing interests.}

The authors have no competing interests

\section{Funding}

The authors pooled funds to support this project from design, data collection, and data analysis through manuscript writing.

\section{Author contributions}

This work was conducted in collaboration between all authors. Authors NT, GK, RN, BA and EK designed the study, wrote the protocol, participated in the data and statistical analysis process, literature searches and wrote the first draft of the manuscript. Author GM did the original conceptualization, supervised the fieldwork laboratory analysis and participated in the write-up of the manuscript. Authors POO and RM participated in the manuscript writing and proof reading. Author LA did the overall supervision of the work right from the development of the protocol, statistical analysis and manuscript conceptualization. All authors read and approved the final manuscript.

\section{References}

1. Oguzkaya-Artan, M., Baykan, Z., Artan, C., \& Avsarogullari L. Prevalence and risk factors for methicillin resistant Staphylococcus aureus carriage among emergency department workers and bacterial contamination on touch surfaces in Erciyes University Hospital, Kayseri, Turkey. African Heal Sci. 2015;15(4):1289-1294.

2. Shibabaw, A., Abebe, T., \& Mihret A. Nasal carriage rate of methicillin resistant Staphylococcus aureus among Dessie Referral Hospital health care workers; Dessie, Northeast Ethiopia. Antimicrob Resist Infect Control. 2013;2(1):25.

3. El Aila, N. A., Al Laham, N. A., \& Ayesh BM. Nasal carriage of methicillin resistant Staphylococcus aureus among health care workers at Al Shifa hospital in Gaza Strip. BMC Infect Dis. 2017;17(1):28.

4. Bazira, J., Boum II, Y., Sempa, J., Iramiot, J., Nanjebe, D., Sewankambo, N., \& Nakanjako D. Trends in antimicrobial resistance of Staphylococcus aureus Isolated from clinical samples at Mbarara 
Regional Referral Hospital in Rural Uganda. Br Microbiol Res J. 2014;4(10):1084.

5. Who. Global priority list of antibiotic-resistant bacteria to guide research, discovery, and development of new antibiotics [Internet]. 2017. Available from: https://www.who.int/.../WHO-PPLShort_Summary_25Feb-ET_NM_WHO.pdf\%0A

6. Daini, O. A., \& Akano SA. Plasmid-mediated antibiotic resistance in Staphylococcus aureus from patients and non patients. Sci Res Essays. 2009;4(4):346-350.

7. Albrich WC1 HS. Health-care workers: source, vector, or victim of MRSA? Lancet Infect Dis. 2008;8(5):289-301.

8. Bagheri Nejad S., B. Allegranzi, S.B. Syed BE and DP. Health-care-associated infections in Africa: a systemic review. World Heal Organ. 2011;89:757-76.

9. Conceiçao, T., Santos Silva, I., de Lencastre, H., \& Aires-de-Sousa M. Staphylococcus aureus nasal carriage among patients and health care workers in São Tomé and Príncipe. Microb Drug Resist. 2014;20(1):57-66.

10. Aiken, A. M., Mutuku, I. M., Sabat, A. J., Akkerboom, V., Mwangi, J., Scott, J. A. G., ... \& Grundmann H. Carriage of Staphylococcus aureus in Thika Level 5 Hospital, Kenya: a cross-sectional study. Antimicrob Resist Infect Control. 2014;3(1):22.

11. Bebell, L. M., Ayebare, A., Boum, Y., Siedner, M. J., Bazira, J., Schiff, S. J., ... \& Firth PG. Prevalence and correlates of MRSA and MSSA nasal carriage at a Ugandan regional referral hospital. J Antimicrob Chemother. 2016;72(3):888-892.

12. Klein, E., Smith, D. L., \& Laxminarayan R. Hospitalizations and deaths caused by methicillin-resistant Staphylococcus aureus, United States, 1999-2005. Emerg Infect Dis. 2007;13(12):1840.

13. WHO. World Health Organization. 2014. Antimicrobial resistance: global report on surveillance. Geneva.

14. Garoy, E.Y., Gebreab, Y.B., Achila, O.O., Tekeste, D.G., Kesete, R., Ghirmay, R., Kiflay, R. and Tesfu T. Methicillin-Resistant Staphylococcus aureus (MRSA): Prevalence and Antimicrobial Sensitivity Pattern among Patients-A Multicenter Study in Asmara, Eritrea. Can J Infect Dis Med Microbiol.

15. Center for Disease Dynamics E\& P. State of the World's antibiotics Washington, DC: Center for Disease Dynamics, Economics \& Policy. 2015.

16. Falagas, M.E., Karageorgopoulos, D.E., Leptidis, J. and Korbila IP. MRSA in Africa: filling the global map of antimicrobial resistance. PLoS One. 2013;8(7):p.e68024.

17. Ojulong, J., Mwambu, T.P., Joloba, M., Bwanga, F. and Kaddu-Mulindwa DH. Relative prevalence of methicilline resistant Staphylococcus aureus and its susceptibility pattern in Mulago Hospital, Kampala, Uganda. Tanzan J Health Res. 2009;11(3).

18. Kateete, D. P., Namazzi, S., Okee, M., Okeng, A., Baluku, H., Musisi, N. L., .. \& Najjuka FC. High prevalence of methicillin resistant Staphylococcus aureus in the surgical units of Mulago hospital in Kampala, Uganda. BMC Res Notes. 2011;4(1):4(1), 326. 
19. Vonberg, R. P., Stamm-Balderjahn, S., Hansen, S., Zuschneid, I., Rüden, H., Behnke, M., \& Gastmeier P. How often do asymptomatic healthcare workers cause methicillin-resistant Staphylococcus aureus outbreaks? A systematic evaluation. Infect Control Hosp Epidemiol. 2006;27(10):1123-7.

20. Dalstrom DJ, Venkatarayappa I, Manternach AL, Palcic MS, Heyse BA PM. Time-dependent contamination of opened sterile operating-room trays. J Bone Jt Surg Am. 2008;90(5):1022-5. pmid:18451394.

21. George, M., Iramiot, J. S., Muhindo, R., Olupot-Olupot, P., \& Nanteza A. Bacterial Aetiology and Antibiotic Susceptibility Profile of Post-Operative Sepsis among Surgical Patients in a Tertiary Hospital in Rural Eastern Uganda. Microbiol Res J Int. 2018;24(2).

22. Clinical and Laboratory Standards Institute. M100 Performance Standards for Antimicrobial Susceptibility Testing, 27th. 2017. 224 p.

23. Aiken, A. M., Mutuku, I. M., Sabat, A. J., Akkerboom, V., Mwangi, J., Scott, J. A. G., ... \& Grundmann H. Carriage of Staphylococcus aureus in Thika Level 5 Hospital, Kenya: a cross-sectional study. Antimicrob Resist Infect Control. 2014;3(1):22.

24. M. Okwu, S. Bamgbala and WA. Prevalence of nasal carriage of community-associated Methicillinresistant Staphylococcus aureus (CA-MRSA) among healthy primary school children in Okada, Nigeria. Prevalence. 2012;2(4):2224-3186.

25. J. Deng, G. Xiao, Y. Zhu, W. Zhou and CW. Staphylococcus aureus nasal carriage and its antibiotic resistance profiles in Tibetan school children in Southwest China. Hong Kong J Pediatr. 2014;19:7578.

26. Ouedraogo, A.S., Dunyach-Remy, C., Kissou, A., Sanou, S., Poda, A., Kyelem, C.G., Solassol, J., Bañuls, A.L., Van De Perre, P. Ouédraogo, R. and Jean-Pierre, H. High nasal carriage rate of Staphylococcus aureus containing panton-valentine leukocidin-and EDIN-encoding genes in community and hospital settings in Burkina Faso. Front Microbiol. 2016;7:p.1406.

27. Moniri, R., Musav, G. A., \& Fadavi N. The prevalence of nasal carriage methicillin-resistant Staphylococcus aureus in hospitalized patients. Pak J Med Sci. 2009;25(4):656-659.

28. Ngoa UA, Schaumburg F, Adegnika AA, Kösters K, Möller T, Gaus E, Fernandes JF, Alabi A, Issifou S, Becker K, Grobusch MP, Kremsner PG LB. Epidemiology and population structure of Staphylococcus aureus in various population groups from a rural and semi urban area in Gabon, Central Africa. Acta Trop. 2012;124(1):42-7.

29. Mehraj J, Akmatov MK, Strömpl J, Gatzemeier A, Layer F, Werner G, Pieper DH, Medina E, Witte W, Pessler F KG. Methicillin-Sensitive and Methicillin-Resistant Staphylococcus aureus Nasal Carriage in a Random Sample of Non-Hospitalized Adult Population in Northern Germany. PLoS One. 2014;9(9):e107937.

30. Tigabu, A., Tiruneh, M., \& Mekonnen F. Nasal Carriage Rate, Antimicrobial Susceptibility Pattern, and Associated Factors of Staphylococcus aureus with Special Emphasis on MRSA among Urban and Rural Elementary School Children in Gondar, Northwest Ethiopia: A Comparative Cross-Sectional Study. Adv Prev Med. 2018; 
31. Moyo SJ, Nkinda L, Majigo M, Mmbaga E, Mbembati N, Aboud S LE. Prevalence of methicillinresistant Staphylococcus aureus carriage on admission among patients attending regional hospitals in Dar es Salaam, Tanzania. BMC Res Notes. 2017;10(1):417.

32. Oyo SJ, Aboud S, Blomberg B, Mkopi N, Kasubi M, Manji K, Lyamuya EF, Maselle SY LN. MHigh nasal carriage of methicillin-resistant Staphylococcus aureus among healthy Tanzanian under- 5 children. Microb drug Resist. 2014;1;20(1):82-8.

33. Mwambi B, Iramiot J, Bwanga F, Nakaye M, Itabangi H, Bazira J. Clindamycin Resistance among Staphylococcus Aureus Isolated at Mbarara Regional Referral Hospital, in South Western Uganda. $\mathrm{Br}$ Microbiol Res J. 2014 Dec 1; 4 (12):1335-1344. Doi: 10.9734/BMRJ/2014/10572. PMID: 26046016; PMCID: PMC4451190.

34. Alsan M, Schoemaker L, Eggleston K, Kammili N, Kolli P BJ. Out-of-pocket health expenditures and antimicrobial resistance in low-income and middle-income countries: an economic analysis. Lancet Infect Dis. 15(10):1203-1210.

\section{Tables}

Table 1: Demographic characteristics of patients attending Mbale RRH. 


\begin{tabular}{|c|c|c|c|}
\hline Demographic factors & Characteristics & Males $(n=64)$ & $\begin{array}{l}\text { Females } \\
(n=154)\end{array}$ \\
\hline Age & $\begin{array}{l}\text { Mean age (40.16; SD } \pm 1 \\
\text { years }\end{array}$ & 17.04) in 45 & 38 \\
\hline \multirow[t]{2}{*}{ Animal contact } & Yes & 42 & 87 \\
\hline & No & 22 & 67 \\
\hline \multirow[t]{2}{*}{ Residence } & Resident in town & 18 & 62 \\
\hline & Resident in village & 46 & 92 \\
\hline \multirow{2}{*}{$\begin{array}{l}\text { History } \\
\text { hospitalization }\end{array}$} & Has ever been hospitalized & 21 & 52 \\
\hline & Never been hospitalized & 42 & 102 \\
\hline \multirow{3}{*}{ Antibiotic usage } & Use antibiotics & 34 & 81 \\
\hline & Does not use antibiotics & 30 & 73 \\
\hline & Negative & 45 & 107 \\
\hline \multirow[t]{3}{*}{ HIV sero-status } & Positive & 5 & 21 \\
\hline & Unknown & 14 & 26 \\
\hline & CoNS & 44 & 72 \\
\hline \multirow[t]{2}{*}{ Isolated organism } & No growth & 9 & 43 \\
\hline & S. aureus & 11 & 39 \\
\hline \multirow{3}{*}{ Methicillin sensitivity } & MRSA & 11 & 25 \\
\hline & MSSA & 0 & 14 \\
\hline & $\mathrm{N} / \mathrm{A}$ & 53 & 114 \\
\hline
\end{tabular}

Key: MRSA- Methicillin Resistant $S$. aureus, MSSA- Methicillin Susceptible $S$. aureus, CoNSCoagulase Negative Staphylococcus, n- proportions per gender

Table 2: Proportions of patients with $S$. aureus nasal carriage.

\begin{tabular}{lll}
\hline Isolated Organism & Frequency (n) & Percentage (\%) \\
\hline S. aureus & 50 & 22.9 \\
CoNS & 116 & 53.2 \\
Not Applicable & 52 & 23.8 \\
\hline Total & 218 & 100 \\
\hline
\end{tabular}

Key: n-number of participants, \%-prevalence rate

Table 3: Proportions of MRSA and MSSA among patients at Mbale RRH.

Key: MRSA-Methicillin Resistant $S$. aureus, MSSA-Methicillin Susceptible $S$. aureus 


\begin{tabular}{|c|c|c|c|c|c|c|c|}
\hline Methicillin Sensitivity & Fre & quency & Per & centage & Cumulative $\mathrm{fr}$ & quency & \\
\hline MRSA & 29 & & 13.3 & & 13.30 & & \\
\hline MSSA & 21 & & 9.63 & & 22.93 & & \\
\hline Not Applicable & 168 & & 77.0 & & 100.00 & & \\
\hline Total & 218 & & 100 & .00 & & & \\
\hline Methicillin Sensitivity & & OR & & $P$-value & 95\% [Confid & ce Inte & val] \\
\hline Age & & $0.9825 \varepsilon$ & 389 & 0.117 & 0.9612351 & 1.0044 & \\
\hline Residence & & 0.71196 & 681 & 0.347 & 0.3505714 & $1.445 !$ & 21 \\
\hline History of Hospitalizatio & & 1.15694 & & 0.687 & 0.5689258 & 2.3527 & 12 \\
\hline Contact with animals & & $1.1410 \varepsilon$ & & 0.455 & 0.8069104 & 1.6136 & \\
\hline Antibiotic use & & $0.7516 \varepsilon$ & 896 & 0.411 & 0.3804775 & $1.485 !$ & \\
\hline $\begin{array}{ll}\text { HIV status } & \text { (Positive) } \\
& \text { (Unknown }\end{array}$ & & $0.7215 \mathrm{c}$ & & 0.551 & 0.2470208 & 2.107 & \\
\hline & & 0.71886 & & 0.481 & 0.2871594 & 1.7995 & 57 \\
\hline
\end{tabular}

nasal colonization.

Key: OR-odds ratios

Table 4: MRSA distribution by disc diffusion and MIC testing .

\begin{tabular}{lcc}
\hline $\begin{array}{l}\text { Methicillin } \\
\text { Sensitivity }\end{array}$ & $\begin{array}{c}\text { Cefoxitin disc screening }(\leq \mathbf{2 1 m m}),(\% \\
[\mathrm{n}])\end{array}$ & $\begin{array}{l}\text { MIC confirmatory } \\
[\mathrm{n}]), \\
\text { Cut-off }(>\mathbf{8} \mathrm{mg} / \mathrm{mL})\end{array}$ \\
\hline MRSA & $72(36)$ & $57.9(29)$ \\
MSSA & $28(14)$ & $42.1(21)$ \\
Total & $100(50)$ & $100(50)$ \\
\hline
\end{tabular}

Key: MRSA-Methicillin Resistant $S$. aureus, MSSA-Methicillin Susceptible $S$. aureus

\section{Figures}




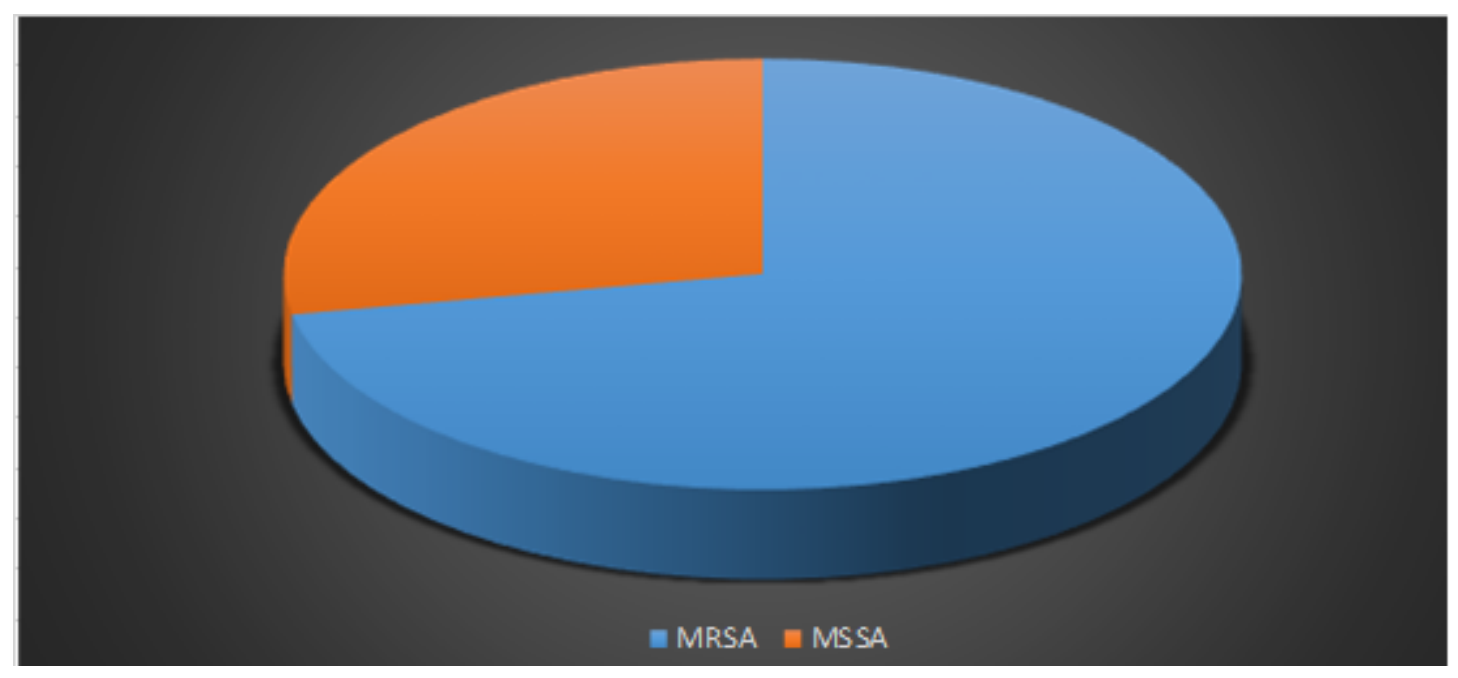

\section{Figure 1}

Distribution of Methicillin Sensitivity among carriers of S. aureus Key: MSSA-Methicillin Susceptible Staphylococcus aureus, MRSA-Methicillin Resistant Staphylococcus aureus 